\title{
An Inflammatory Fibroid Polyp (Vanek's Tumor) of the Ileum that Presented as Intussusception
}

\author{
Jung-Hee Yoon \\ Department of Radiology, Inje University Haeundae Paik Hospital, College of Medicine, Busan, Republic of Korea \\ Email: radyjh@hanmail.net
}

Received November 19, 2013; revised December 15, 2013; accepted December 20, 2013

Copyright (C) 2013 Jung-Hee Yoon. This is an open access article distributed under the Creative Commons Attribution License, which permits unrestricted use, distribution, and reproduction in any medium, provided the original work is properly cited.

\begin{abstract}
Ileoileal intussusceptions caused by inflammatory fibroid polyps are uncommon. Inflammatory fibroid polyps (Vanek's tumor) are rare benign lesions that originate in the submucosa of the gastrointestinal tract. Here, we report the case of a 54-year-old woman who was admitted with an acute onset of abdominal pain and signs of intestinal obstruction. The ileal segment was resected, and single-layer end-to-end anastomosis was performed. A submucosal polypoid mass measuring approximately $4.5 \times 4.0 \mathrm{~cm}$ was found. The histopathological analysis revealed an ulcerated mucosal lesion with variable cellularity that was formed by spindle cells with slight mitosis and abundant inflammatory infiltrate that was mainly composed of eosinophils. Immunohistochemistry confirmed the diagnosis of ileal inflammatory fibroid polyps. Cross-sectional imaging (multidetector computed tomography) plays a significant role in diagnosing this condition and guiding its management.
\end{abstract}

Keywords: Intussusception; Intestinal Obstruction; Intestinal Polyp; Computed Tomography; Gastrointestinal Neoplasms; Diagnosis

\section{Introduction}

Intestinal intussusceptions are relatively common causes of intestinal obstruction that occur most frequently in the ileocolic area. Benign disease is the typical cause in children and adolescents, while malignant or adhesive diseases are far more common in older patients. Cases of adult intussusceptions account for only $1 \%-5 \%$ of all small bowel obstructions [1]. Adenocarcinoma is the most common cause of intussusceptions in adults. Inflammatory fibroid polyps are rare, non-neoplastic lesions that originate in the submucosa of the gastrointestinal tract. They were first described in the stomach by Vanek in 1949 [2]. Inflammatory fibroid polyps are benign, non-encapsulated lesions that are mainly comprised of loose connective tissues and vessels, with eosinophilic inflammatory components [3].

The present report describes the case of a patient who presented with intussusceptions and intestinal obstruction caused by an ileal inflammatory fibroid polyp. Inflammatory fibroid polyps are rarely seen in adults, but they are among the probable diagnoses that should be considered in obstructive tumors of the small bowel causing intussusceptions.

\section{Case Description}

A 54-year-old woman presented at the emergency department in our hospital with sudden right upper quadrant and chest pain, respiratory difficulty, and a 3-day history of fever, chills, nausea, and vomiting. During the physical examination, the patient's abdomen was mildly tender and distended, but there was no palpable mass in the abdomen. Her blood pressure was 100/70 $\mathrm{mmHg}$, and her pulse was 78 beats/min. The peripheral blood examination results were white blood cell $10,370 \mathrm{~mm}^{3}$, red blood cell $12.6 \mathrm{mg} / \mathrm{dL}$, platelet count $113,000 \mathrm{~mm}^{3}$, and c-reactive protein $0.06 \mathrm{mg} / \mathrm{dL}$. The liver function test was unremarkable. The patient had another additional episode of abdominal pain three months prior, and acute pancreatitis was suspected. The patient had no history of anemia or gastrointestinal bleeding.

Abdominal multidetector computed tomography (CT) demonstrated diffuse distention of ileal loops and luminal narrowing in the distal ileum with ileoileal intussusceptions (Figures 1(A) and (C)), mesenteric edema, and multiple enlarged mesenteric lymph nodes. An abnormal, large, soft tissue intraluminal mass measuring $4.5 \times 4.0$ $\mathrm{cm}$ was noted in the distal ileum and in the head of the 
intussusceptions attached by a stalk (Figures 1(B) and (D)). The soft tissue mass was presumed to be the leading point of the intussusceptions. The clinical impression was that of gastrointestinal stromal tumor (GIST), lymphoma, inflammatory fibroid polyp, adenomatous polyp, or metastasis. We believed that the possibility of adenocarcinoma of the small bowel was less likely because a spherical low attenuation soft tissue mass demonstrated as delayed with mild enhancement, and a long enhancing central fibrovascular stalk was noted on the coronal reconstruction multidetector CT image (Figure 1(D)). Prominent enhancement of the normal overlying ileal mucosa suggests that the tumor originated from the subepithelium and not from the epithelium.

An exploratory laparotomy was performed, and an ileoileal intussusception was found, with a lesion measuring approximately $50 \mathrm{~cm}$ depart from the ileocecal valve. A segmental resection of the affected ileum was performed, followed by single-layer end-to-end anastomosis. The submucosal polypoid mass (Figure 2) measured approximately $4.5 \times 4.0 \mathrm{~cm}$, and the histological report showed that the ileal tumor had variable cellularity and was formed by spindle cells, with slight mitosis and abundant vascular and fibroblastic proliferation with inflammatory infiltrate that was mainly composed of eosinophils (Figure 3). In the immunohistochemical studies, the tumors were diffusely immunoreactive for actin, vimentin, CD 68, CD 34 (Figure 4), and platelet-derived growth factor receptor-alpha and negative for C-kit (CD 117), and S-100 protein. Based on these pathologic and

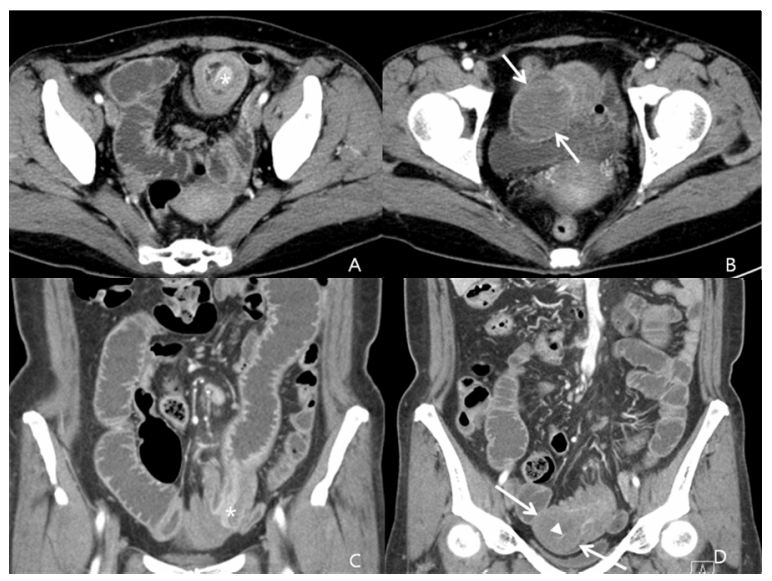

Figure 1. A 54-year-old woman with a surgically diagnosed inflammatory fibroid polyp of the ileum. (A), (B) An axial enhanced CT section shows small bowel intussusception loops in the pelvis and a soft tissue mass at the head of the intussusception (arrows). Prominent enhancement of the normal overlying ileal mucosa (asterisk) suggests that the tumor originated from the subepithelium. (C), (D) A spherical low-attenuation soft tissue mass (arrows) with delayed mild enhancement and with a long enhancing central fibrovascular stalk (arrowhead) was noted on the coronal reconstruction multidetector CT image.

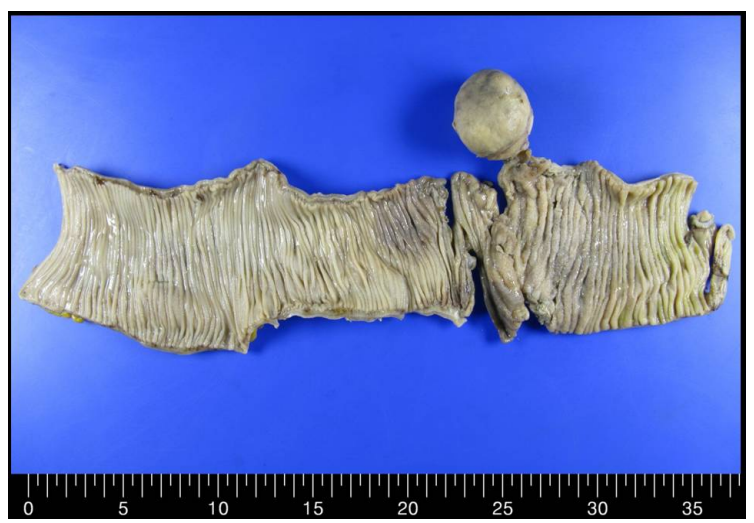

Figure 2. The resected ileum with an inflammatory fibroid polyp. This submucosal polypoid mass measured approximately $4.5 \times 4.0 \mathrm{~cm}$.

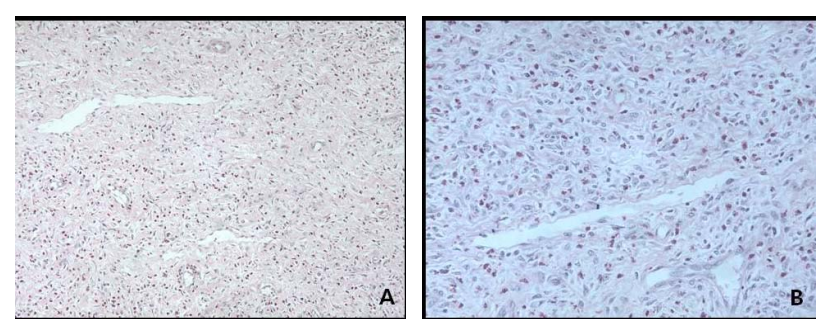

Figure 3. Histopathology (hematoxylin and eosin stain, A, $\times 200$ magnification) revealed that the tumor had variable cellularity and abundant vascular structures $(B, \times 400$ magnification) formed by spindle cells with slight mitosis and fibroblastic proliferation with inflammatory infiltrate that was mainly composed of eosinophils.

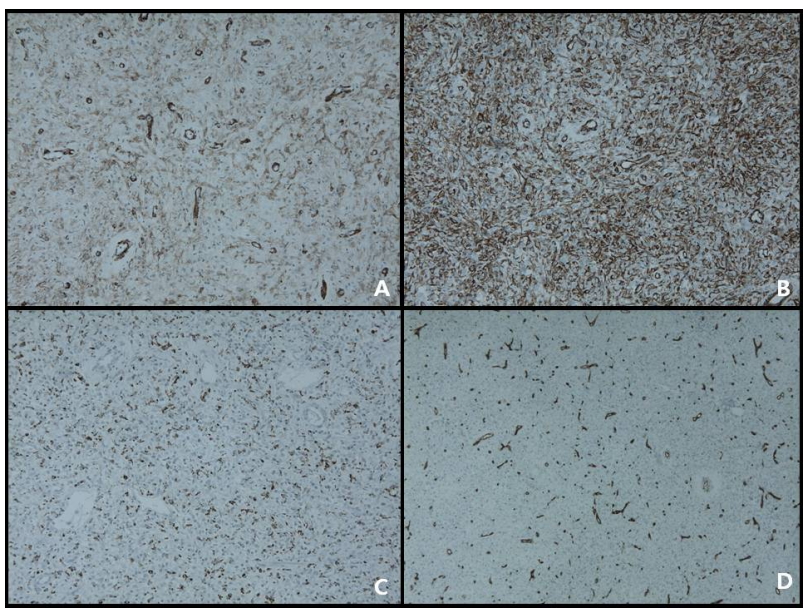

Figure 4. Immunohistochemically, the tumor cells are weak positive for smooth muscle actin (A, $\times 100$ magnification), strong positive for vimentin $(B, \times 100$ magnification), positive for CD 68 (C, $\times 100$ magnification), and positive to thin vessels only for CD 34 (D, ×100 magnification).

immunohistochemical findings, a diagnosis of ileal inflammatory fibroid polyps was made. The patient had an uneventful postoperative course over the following 6 months. 


\section{Discussion}

Inflammatory fibroid polyps (Vanek's tumor) are rare benign non-neoplastic lesions of the gastrointestinal tract that were first described in the literature as "gastric submucosal granuloma with eosinophilic infiltration" in a study by Vanek in 1949 (2).

They later became recognized under a variety of different names, such as inflammatory pseudotumor, granuloma with eosinophils, eosinophilic granuloma, and polyp with eosinophilic granuloma. Four years after initially appearing in the literature, the term "inflammatory fibroid polyp" was introduced in a study by Helwig et al., and it has become the most widely used term [4].

Inflammatory fibroid polyps can develop in many different locations in the gastrointestinal tract. They are primarily found in the gastric antrum $(70 \%)$ or in the ileum $(20 \%)$ and, rarely, in the duodenum and jejunum [5]. Inflammatory fibroid polyps usually measure 2 - 5 $\mathrm{cm}$ in diameter. However, giant inflammatory fibroid polyps measuring up to $12.5 \mathrm{~cm}$ in diameter have been reported [6]. Inflammatory fibroid polyps are usually found incidentally in asymptomatic patients [7]. However, patients can also present with abdominal pain, weight loss, bleeding, dyspeptic symptoms and obstruction. Symptoms are dependent on the site and size of the lesion [8].

The present case is one that is rarely reported in the literature; the intestinal obstruction was caused by ileoileal inflammatory fibroid polyp intussusceptions. Sixty-five percent of all adult intussusceptions occur because of a malignant or a benign lesion, usually appearing at the head of the invagination [9]. Malignant causes are found in $43 \%$ to $80 \%$ of intussusceptions located in the colon compared to approximately $14 \%$ to $47 \%$ of cases occurring in the small intestine. Although cases of adult intussusceptions caused by benign processes are rare, benign tumors have been found as the lead points of intussusceptions and have also been associated with lipomas, leiomyomas, neurofibromas, adenomas and, rarely, inflammatory fibroid polyps [1].

In a case series of 75 patients presenting with inflammatory fibroid polyps in the gastrointestinal area, Johnstone et al. reported that $75 \%$ of all cases were gastric inflammatory fibroid polyps [10]. The occurrence of inflammatory fibroid polyps in the small intestines is, accounting for only $18 \%$ of all inflammatory fibroid polyp cases [10]. A recent meta-analysis found 86 published (in the English literature) cases of intussusception caused by inflammatory fibroid polyps, but CT imaging analysis was rarely performed, only 26 cases were described ([11]. It has a similar incidence in both sexes, and the patients were aged from 4 to 81 years (mean, $49 \pm 16.2$ years).

According to the localization of inflammatory fibroid polyps, 64 patients had ileal intussusceptions, while 17 had jejunal, three had colonic, and two had ileojejunal intussusceptions. The current case is one of the few reported in the literature in which the intussusceptions are caused by a terminal ileal inflammatory fibroid polyp.

Inflammatory fibroid polyps can mimic several other tumor and non-tumor processes in the gastrointestinal tract. The differential diagnoses include spindle cell lesions, such as gastrointestinal stromal tumors (GISTs), spindle-cell carcinoids and inflammatory fibrosarcoma in the histopathology. Differentiation can be difficult, particularly between inflammatory fibroid polyps and GISTs [12]. There were no distinctive features to differentiate inflammatory fibroid polyps from other subepithelial lesions of the gastrointestinal tract. GISTs are common in the stomach and frequently present as polypoid masses. In the intestine, these tumors can present with intussusceptions that are similar to inflammatory fibroid polyps. Immunohistochemistry is used to distinguish between inflammatory fibroid polyps and GISTs. Both tumors are positive for CD34 and vimentin, but GISTs are positive for C-kit (CD 117), while inflammatory fibroid polyps are not [13].

The majority of intussusceptions are only diagnosed during surgery. The preoperative diagnosis of intussusceptions, which was performed with imaging techniques in the present case, is rare. Ultrasound is the primary imaging modality of choice or the first-line examination for intussusception, and the classic imaging features include the target, bull's eye or doughnut sign in the transverse view and the pseudokidney, sandwich, or hayfork sign in the longitudinal view [14].

Ultrasonography enables the diagnosis or exclusion of intussusceptions with a sensitivity of $97.9 \%$ to $98.5 \%$, a specificity of $97.8 \%$ to $100 \%$, a positive predictive value of $86.6 \%$ and a negative predictive value of $99.7 \%$ $[15,16]$. However, obesity and the presence of a large amount of air in the distended bowel loops can limit the image quality and diagnostic accuracy. CT imaging from a group of 136 adult intussusceptions was used to diagnose surgical enteroenteric intussusceptions. Using the criteria of length $>3.5 \mathrm{~cm}$, the imaging diagnosis yielded a mean sensitivity of $100 \%$ and a specificity of $57.3 \%$. Using a measured axial diameter $>3 \mathrm{~cm}$, the mean sensitivity and specificity were $100 \%$ and $32.9 \%$, respectively [17]. On CT, a bowel-within-bowel configuration, which is suggested by mesenteric fat and vessels compressed between the walls of the small bowel, is pathognomonic of intussusceptions [18]. In contrast to ultrasonography, CT is unaffected by the presence of gas in the bowel lumen. Therefore, we suggest that all patients presenting with an intestinal obstruction should undergo a CT scan as a routine diagnostic tool.

The etiology and pathogenesis of inflammatory fibroid 
polyps remains unknown, but it could be a consequence of chronic irritation and inflammation or a consequence of an extreme body reaction to an intestinal trauma or a localized variant of eosinophilic gastroenteritis, given that it has marked eosinophilic infiltration [19].

Based on the histological examination alone, some authors initially believed that inflammatory fibroid polyps were a true neoplasm of either neural or vascular origin.

However, immunohistochemical studies refuted this possibility after negative staining for S100 protein and factor VIII-related antigen in proliferating cells was demonstrated [20]. According to these different immunostaining patterns, there is the possibility of a span of morphological and immunohistochemical patterns indicating different phases of inflammatory reaction [13]. Exploratory laparotomy is frequently recommended as the best treatment for intussusceptions caused by inflammatory fibroid polyps. The lesions seem to lack malignant potential, and polyp recurrence has only been reported once [21].

The appropriate management of adult intussusceptions remains controversial, with the debate mainly focused on the issue of primary en bloc resection versus initial reduction followed by more limited resection. Theoretically, surgical reduction before resection may permit more limited resection; however, the risk of potential intraluminal seeding or venous tumor dissemination during the manipulation of a malignant lesion should also be considered. The incidence of malignancy as the cause of small intestinal intussusceptions ranges from $1 \%$ to $47 \%$, and the majority of lesions are metastatic. Therefore, recent reports have recommended the initial reduction of externally viable small bowel prior to resection. The likelihood of cancer in ileocolic and colocolic intussusceptions is $43 \%$ - $100 \%$. The vast majority of these lesions arise as a primary lesion, for which resection without reduction is recommended [11]. The surgery should be performed as early as possible to prevent the intussusceptions from leading to ischemia or necrosis and the subsequent perforation of the invaginated bowel segment. When surgery is delayed and intestinal perforation with peritonitis occurs, there is a considerable increase in morbidity and mortality. Fortunately, the patient in this report, despite undergoing emergency surgery, recovered satisfactorily and was discharged after 6 days, with no signs of recurrence 6 months after surgery.

In conclusion, we reported a rare case of an inflammatory fibroid polyp (Vanek's tumor) of the ileum that presented as intussusception. Intussusception is a rare cause of adult intestinal obstruction, and inflammatory fibroid polyp is one of the least common causes of this rare condition. We also described the proper treatment of this condition.

\section{REFERENCES}

[1] A. Karamercan, O. Kurukahvecioglu, T. U. Yilmaz, G. Aygencel, B. Aytac and M. Sare, "Adult Ileal Intussusceptions: An Unusual Emergency Condition,” Advances in Therapy, Vol. 23, No. 1, 2006, pp. 163-168. http://dx.doi.org/10.1007/BF02850357

[2] J. Vanek, "Gastric Submucosal Granuloma with Eosinophilic Infiltration,” American Journal of Pathology, Vol. 25, No. 3, 1949, pp. 397-411.

[3] C. Ng, K. Y. Lam, T. S. Gupta and Y. H. Ho, "Inflammatory Fibroid Polyp of the Caecum in a Patient with Neurofibromatosis,” ANNALS Academy of Medicine Singapore, Vol. 33, No. 6, 2004, pp. 797-799.

[4] K. A. Malik, G. K. Pande, Z. Aftab and V. Nirmala, "Inflammatory Fibroid Polyp of the Ileum Causing Intussusceptions,” Saudi Medical Journal, Vol. 26, No. 6, 2005, pp. 995-998.

[5] A. P. Wysocki, G. Taylor and J. A. Windsor, "Inflammatory Fibroid Polyps of the Duodenum: A Review of the Literature,” Digestive Surgery, Vo. 24, No. 3, 2007, pp. 162-168. http://dx.doi.org/10.1159/000102099

[6] R. K. Harned, J. L. Buck and K. M. Shekitka, "Inflammatory Fibroid Polyps of the Gastrointestinal Tract: Radiologic Evaluation,” Radiology, Vol. 182, No. 3, 1992, pp. 863-866.

[7] D. Paikos, J. Moschos, D. Tzilves, A. Koulaouzidis, G. Kouklakis, F. Patakiouta, K. Kontodimou, A. Tarpagos and I. Katsos, "Inflammatory Fibroid Polyp or Vanek's tumour,” Digestive Surgery, Vol. 24, No. 3, 2007, pp. 231-233. http://dx.doi.org/10.1159/000103326

[8] A. P. Wysocki, G. Taylor and J. A. Windsor, "Inflammatory Fibroid Polyps of the Duodenum: A Review of the Literature,” Digestive Surgery, Vol. 24, No. 3, 2007, pp. 162-168. http://dx.doi.org/10.1159/000102099

[9] N. K. Nkanza, M. King and M. S. R. Hutt, "Intussusception Due to Inflammatory Fibroid Polyps of the Ileum: A Report of 12 Cases from Africa," British Journal of Surgery, Vol. 67, No. 4, 1980, pp. 271-274. http://dx.doi.org/10.1002/bjs.1800670414

[10] J. M. Johnstone and B. C. Morson, "Inflammatory Fibroid Polyp of the Gastrointestinal Tract," Histopathology, Vol. 2, No. 5, 1978, pp. 349-361. http://dx.doi.org/10.1111/j.1365-2559.1978.tb01727.x

[11] S. Akbulut, "Intussusception Due to Inflammatory Fibroid Polyp: A Case Report and Comprehensive Literature Review,” World Journal of Gastroenterology, Vol. 18, No. 40, 2012, pp. 5745-5752.

http://dx.doi.org/10.3748/wjg.v18.i40.5745

[12] B. Coulier, P. Maldaque, B. Broze and I. Gielen, "Ileal Inflammatory Fibroid Polyp Causing Ileocolic Intussusceptions,” JBR-BTR, Vol. 91, No. 4, 2008, pp. 149-152.

[13] S. O. Cawich, T. N. Gibson, D. I. Mitchell, E. Williams, M. S. Newnham and L. K. Simpson, "Adult Intussusceptions from an Inflammatory Fibroid Polyp: A Case and Review of the Literature," The Internet Journal of Pathology, Vol. 7, 2008, p. 1.

[14] S. Yakan, C. Caliskan, O. Makay, A. G. Denecli and M. A. Korkut, "Intussusception in Adults: Clinical Charac- 
teristics, Diagnosis and Operative Strategies," World Journal of Gastroenterology, Vol. 15, No. 16, 2009, pp. 1985-1989. http://dx.doi.org/10.3748/wig.15.1985

[15] A. L. Hryhorczuk and P. J. Strouse, "Validation of US as a First-Line Diagnostic Test for Assessment of Pediatric Ileocolic Intussusceptions,” Pediatric Radiology, Vol. 39, No. 10, 2009, pp.1075-1079. http://dx.doi.org/10.1007/s00247-009-1353-z

[16] R. L. Shanbhogue, S. M. Hussain, M. Meradji, S. G. Robben, J. E. Vernooij and J. C. Molenaar, "Ultrasonography Is Accurate Enough for the Diagnosis of Intussusceptions,” Journal of Pediatric Surgery, Vol. 29, No. 2, 1994, pp. 324-327.

[17] B. Sundaram, C. N. Miller, R. H. Cohan, M. J. Schipper and I. R. Francis, "Can CT features Be Used to Diagnose surgical Adult Bowel Intussusceptions?” American Journal of Roentgenology, Vol. 193, No. 2, 2009, pp. 471-478. http://dx.doi.org/10.2214/AJR.08.1801

[18] A. Marinis, A. Yiallourou, L. Samanides, N. Dafnios, G. Anastasopoulos, I. Vassiliou and T. Theodosopoulos, "Intussusception of the Bowel in Adults: A Review," World
Journal of Gastroenterology, Vol. 15, No. 4, 2009, pp. 407-411. http://dx.doi.org/10.3748/wjg.15.407

[19] M. Barussaud, N. Regenet, X. Briennon, B. de Kerviler, P. Pessaux, N. Kohneh-Sharhi, P. A. Lehur, A. Hamy, J. Leborgne, J. C. le Neel and E. Mirallie, "Clinical Spectrum and Surgical Approach of Adult Intussusceptions: A Multicentric Study,” International Journal of Colorectal Disease, Vol. 21, No. 8, 2006, pp. 834-839. http://dx.doi.org/10.1007/s00384-005-0789-3

[20] J. J. Navas-Palacios, F. Colina-Ruizdelgado, M. D. Sanchez Larrea and J. Cortes Causino, "Inflammatory Fibroid Polyp of the Gastrointestinal Tract: An Immunohistochemical and Electron Microscopic Study,” Cancer, Vol. 51, No. 9, 1983, pp. 1682-1690. http://dx.doi.org/10.1002/1097-0142(19830501)51:9<168 2::AID-CNCR2820510921>3.0.CO;2-P

[21] C. Santos Gda, V. A. F. Alves, A. Wakamatsu and S. Zucoloto, "Inflammatory Fibroid Polyp, an Immunohistochemical Study,” Arquivos de Gastroenterologia, Vol. 41, No. 2, 2004, pp. 104-107.

\section{Abbreviation}

CT: Computed Tomography

GISTs: gastrointestinal stromal tumors 